Piskin, S. et al: Computational modeling of neonatal cardiopulmonary bypass

\title{
Computational modeling of neonatal cardiopulmonary bypass hemodynamics with full Circle of Willis anatomy
}

\author{
Senol Piskin ${ }^{1}$, Akif Undar ${ }^{2}$, and Kerem Pekkan ${ }^{1,3}$ \\ ${ }^{1}$ Department of Mechanical Engineering, Koc University, Sariyer, Istanbul, Turkey \\ ${ }^{2}$ Pediatric Cardiovascular Research Center, Department of Pediatrics, Surgery and \\ Bioengineering, Penn State Hershey College of Medicine, Hershey, PA, USA \\ ${ }^{3}$ Department of Biomedical Engineering, Carnegie Mellon University, Pittsburgh, PA, USA
}

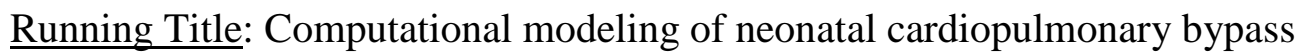

This study is partially presented at the 10th International Conference on Pediatric Mechanical Circulatory Support Systems and Pediatric Cardiopulmonary Perfusion, May 28 - 31, 2014 The Hall of Flags, University of Pennsylvania

Address for Correspondence:

Kerem Pekkan, PhD.

Associate Professor - Biomedical \& Mechanical Engineering

Carnegie Mellon University

700 Technology Dr, Pittsburgh, PA 15219

Phone: (412) 2683027

Fax: (404) 2689807

e-mail:kpekkan@andrew.cmu.edu 
Piskin, S. et al: Computational modeling of neonatal cardiopulmonary bypass

\section{Abstract}

Cardiopulmonary bypass ( $\mathrm{CPB}$ ) procedure is employed to repair most of the congenital heart defects (CHD). Cannulation is a critical component of this procedure where the location and diameter of cannula controls the hemodynamic performance. State-of-the-art computational studies of neonatal $\mathrm{CPB}$ employed an isolated aortic arch region by truncating the threedimensional (3D) patient-specific cerebral system. Present work expanded these studies where the 3D patient-specific MRI reconstruction of the cerebral system, including the Circle of Willis $(\mathrm{CoW})$, is integrated with a hypoplastic neonatal aortic arch. Inlet of the arterial cannula is assigned a steady velocity boundary condition of the CPB pump, while all outlets are modeled as resistance boundary conditions, thus allowing acute comparisons between different cannula configurations. 3D Flow simulations in the aortic arch model are performed at a Reynolds number of 2150 using an experimentally validated commercial solver. Results demonstrate that the inclusion of $3 \mathrm{D} \mathrm{CoW}$ is essential to predict the accurate head-neck blood perfusion and therefore detrimental in deciding the neonatal aortic cannulation strategy pre-operatively. Using this integrated model two CPB configurations are studied, where the cannulas were placed at Innominate Artery (IA-cannula configuration) and Ductus Arteriosus (DA-cannula configuration). Configuration change produced significant differences in flow splits and local hemodynamics of blood flow throughout the whole aortic arch, neck and cerebral arteries. Percent flow rate differences between the IA- and DA-cannula configurations are computed to be: 19, for descending aorta, 198 for ascending aorta (perfusing coronary arteries), 91\% for right anterior cerebral artery, and $68 \%$ for left anterior cerebral artery. Another important finding is the retrograde flow at vertebral arteries for IA-cannula configuration, but not for DA-cannula. These results may help to translate better neonatal arterial cannulae design for minimizing cerebral complications during CPB procedures.

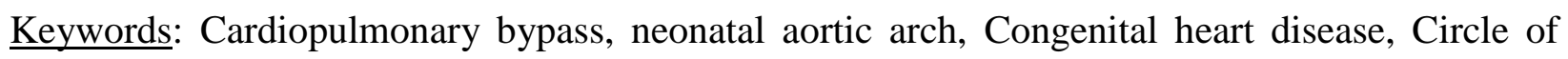
Willis, thrombus, blood flow, vortex, wall shear stress, Fluent, CFD validation 
Piskin, S. et al: Computational modeling of neonatal cardiopulmonary bypass

\section{Introduction}

Despite the advances in surgical techniques and medical management, congenital heart defects still have a significant impact on morbidity, mortality, and healthcare costs (Ref-STS database). In particular, neonates (0-30 days) have the highest mortality rate of $10.2 \%$ compared to all other age groups. Successful surgical repair of congenital heart defects depend on optimal neonatal cardiopulmonary bypass procedure (CPB). A poor CPB procedure can lead to cerebral complications and developmental defects. This is especially true in neonates, where their fragility leads very little room for error. Neonatal CPB requires tiny aortic cannulae (1.2 - $2 \mathrm{~mm}$ inner diameter), with micro-scale blood-wetting features to deliver relatively large blood volumes $(0.3-0.8 \mathrm{~L} / \mathrm{min})$ resulting in extremely high-blood flow velocities. These severe flow conditions initiate platelet activation, release inflammatory cytokines, and further result vascular and blood damage. To control these adverse flow regimes, novel diffuser-tipped aortic cannulae geometries with internal helical contours have been introduced by our group [1] and reported their superior tip shape sensitivity to cerebral perfusion. Most recently similar internal flow features are also considered for improving the adult CPB cannula design [2, 3]. Reduced cerebral embolism is desired for adult and neonatal CPT applications [4]. This is realized through backward suction cannula tips for adults but it is not possible for neonatal patients [5]. Vacuum assisted venous drainage, pump flow rate, hct levels along with cardiotomy suction have an impact on microemboli generation. For neonatal CPB the quality of cerebral perfusion is more critical and focused in the present study.

Cerebral perfusion and hemodynamics during CPB has been studied extensively through bioengineering approaches both for neonatal $[1,6]$ and adult patients $[7,8]$. Pekkan et al [9] reported the detailed three-dimensional (3D) pulsatile hemodynamics, mechanical loading, and perfusion characteristics of a patient-specific neonatal aortic arch during CPB 
Piskin, S. et al: Computational modeling of neonatal cardiopulmonary bypass

using an experimentally validated cross-clamped aortic arch computational fluid dynamics (CFD) model. Zelicourt et al [10] extended this study to investigate the effect of different cannulation strategies in neonates using idealized aortic arch templates of congenital heart defects. Lumped parameter models (LPM) of CPB circuit are first proposed to study cerebral auto-regulation in adult CPB [11]. This modeling feature is not valid for neonatal CPB since pressure-flow autoregulation is lost during neonatal $\mathrm{CPB}$ as the mean arterial pressure is kept low at $30 \mathrm{mmHg}$ and pump flow rates reach 150 to $200 \mathrm{ml} / \mathrm{kg} / \mathrm{min}$. Kaufmann et al. [12] integrated LPM models with CFD, performed basic in vitro mock-up loop experiments for validation leading to improved peripheral flow modeling [13].

These modeling studies provided important information on the mechanics of cerebral perfusion but they all lack the unique functional features of the complex cerebral arterial system, such as the Circle of Willis (CoW), since in almost every model the cerebral arteries are treated as a single lumped resistance elements attached downstream of the head-neck vessels. CoW system regulates the blood flow in the brain by allowing the transition of blood from posterior cerebral arteries to anterior cerebral arteries through its special vascular network. While this modeling limitation may be a lesser concern for adults, the peripheral resistance values for neonatal patients are comparatively higher and determine the integrated physics of the neonatal arch and cerebral artery system by fixing the head-neck and descending aorta flow splits. As such, the computational models that focused only the aortic arch region $[1,2]$ paradoxically did not fully confirmed the clinical experience that indicated the importance of aortic cannula configuration for improved multi-organ perfusion, possibly due to the very high peripheral bed resistance values of the neonatal patient [2]. Therefore in this manuscript, to confirm this hypothesis, an extended neonatal arch model is developed that includes the main cerebral great vessels, including the CoW and the Basal Ganglia. 
Piskin, S. et al: Computational modeling of neonatal cardiopulmonary bypass

These arterial vessels are known to be critical due to their major role in blood flow redistribution, backflow and cerebral CPB perfusion $[14,15]$.

During the last decade, intraoperative cerebral flow monitoring became a standard surgical tool and utilized widely [16-19]. A number of real-time intraoperative imaging modalities and systems have been proposed to improve the surgical outcome (such as NIRS, EVAC etc) [20-22]. Understanding flow structures and hemodynamics in cerebral great arteries is important for accurate and more meaningful intraoperative imaging of cerebral perfusion and blood flow.

Present manuscript investigates the effect of cannula configuration on the flow distributions and hemodynamic properties of cerebral great arteries. Hypoplastic aortic arch is selected as a standard congenital neonatal aortic arch template. In addition, hypoloastic left heart syndrome is a very complex and high-risk model, so more complications of all vital organs but in particular in the brain, are expected. Using this anatomy, cerebral blood flow distributions of two different cannula configurations were compared. Adaptation of quantitative bioengineering principles with an emphasis on aortic cannulation and discovery of quantitative multi-organ damage indices through predictive quantitative computational models will improve the hemodynamic performance of neonatal CPB procedure.

\section{Materials and Methods}

\subsection{Anatomy of aortic arch cannulation, head-neck and cerebral arteries}

In order to be consistent with earlier investigations the realistic 3D hypoplastic aortic arch model is utilized as in our previous studies $[9,10]$. This complex arch model include both ascending aorta (Aao) and main pulmonary artery (MPA) and five outlets; descending aorta (Dao), right pulmonary artery (RPA), left pulmonary artery (LPA) and three neck arteries; IA, left carotid artery (LCA) and left subclavian artery (SA). This model incorporates a fully 
Piskin, S. et al: Computational modeling of neonatal cardiopulmonary bypass

open DA which is connected to the transverse arch at istmus. For complete abbreviations of the vessels please refer to Table 1 . This arch geometry is integrated with the cerebral arterial system, which includes major cerebral arteries and CoW region (Figure 1). Cerebral computer tomography images of neonatal subjects are not ethically justified and an IRB approved patient-specific MRI scans of a healthy young adult is utilized and found to be sufficient for the present investigation. The 3D patient-specific cerebral reconstruction was scaled-down so that the dimensions of the arteries are matched to the neonatal dimensions (Figure 1). All geometries were coupled and post-processed using Geomagic (Geomagic Inc., NC, USA) and our in house anatomical editing software [23].

There exists two common cannula insertion locations $[9,10]$ and four configurations investigated previously [10]. Based on these earlier results, in the present study we selected the configurations that showed the largest local hemodynamic differences even though the previous computational results did not show a substantial change in flow distributions [10]. Two common neonatal cannula configurations that are commonly employed for $1^{\text {st }}$ stage arch reconstructions are introduced to the integrated cerebral and aortic model. The locations of the cannulas are the same as our previous study [10] to enable comparison. A generic cannula is considered with a diameter of $4 \mathrm{~mm}$ as in our earlier work. First cannula configuration is introduced from the Aao-IA junction, while the second one simulates a ductal cannula (DA). Those two cannula configurations are referred as the IA-cannula and DA-cannula throughout this manuscript, (Figure1).

\subsection{Resistance boundary conditions}

Both the isolated aorta geometry as well as the integrated model has resistance boundary conditions imposed at the outlets. Isolated geometry's peripheral resistances are specified at the outlet vessels of Dao, RPA, LPA, IA, LCA, and SA while the integrated model has the 
Piskin, S. et al: Computational modeling of neonatal cardiopulmonary bypass

peripheral resistances at Dao, RPA; LPA, SA, RACA, LACA, RMCA, LMCA, RPCA, and LPCA outflow boundaries. Patient-specific neonatal resistance values utilized in this study are same as in our previous isolated aorta geometry [10] that are obtained by matching physiological flow distributions for neonates. These resistance values are 2,370, 5,923, 3,933, 1,189, and 2,740 MPa.s.m ${ }^{-3}$ for IA, LCA, SA, Dao and RPA and LPA, respectively which are sufficiently reduced to represent the additional CoW segment. For the new integrated model this small reduction ensured the same total systemic and cerebral flow rates for both models. In the new integrated model there are totally 2 inlets and 10 outlets; 6 at cerebral arteries and 3 at aortic arch (Figure 1).

\subsection{Computational fluid dynamics}

The Ansys Mesher 15.0 (Ansys, Inc., PA, USA) grid generation package was used to generate the 3D mesh. Assessment of the mesh quality was defined as devoid of high cell skewness, as there is a known relationship between mesh quality, simulation convergence, and convergence time [24].

As a CFD solver, FLUENT 15.0 (Ansys, Inc., PA, USA) was selected incorporating our previous cardiovascular CFD models [9, 23, 25-27]. Briefly this CFD code utilizes a multi-grid artificial compressibility package for incompressible, Newtonian flows, and employs a second-order accurate numerical discretization scheme. The simulation used a high-density spatial grid with a total of $\sim 1 \mathrm{M}$ fluid nodes, with a grid spacing of $0.7 \mathrm{~mm}$, and a simulation time step of $1 \times 10^{-5}$ seconds in physical time to achieve the resolution required to converge with the mesh size. A fully-developed velocity profile is applied at the inlets corresponding to a steady cannula inlet flow rate of $0.525 \mathrm{~L} / \mathrm{min}$. Simulations continued until convergence of $10^{-3}$ residue as an absolute error. The conservation of mass criterion was checked for verification of all models. Difference between inflow and total outflow is less than $10^{-8} \mathrm{~L} / \mathrm{min}$. indicating that the total mass is conserved in simulations. 
Piskin, S. et al: Computational modeling of neonatal cardiopulmonary bypass

\subsection{Mesh Convergence Tests}

A mesh density sensitivity campaign is conducted [28] based on achieving a relative difference of less than $5 \%$ variations in velocity profile at Dao region just downstream of the DA connection (Figure 2). The geometry was discretized for 6 different element sizes (starting with $1.3 \mathrm{~mm}$ node size, to $0.5 \mathrm{~mm}$ ). Grid sensitivity analysis was conducted using grids of increasing mesh sizes. The element size versus total number of elements in the coupled geometry is shown in Table 2. There are 6 different mesh sizes and they are given in Table 2 to specify number of elements. 5 cases for 5 different mesh sizes were run and resulting velocity profiles are shown in Figure 2. $6^{\text {th }}$ mesh size result is not included in the figure since the mesh convergence is obtained with 5 cases. Figure 2 shows that the velocity profile has an acceptable convergence for mesh sizes smaller than $0.7 \mathrm{~mm}$.

\section{Results}

In this section, first the isolated aorta geometry and the integrated aorta-CoW result will be compared, followed by the simulation results of the two neonatal cannula configurations, Beginning with the differences in the basic flow structures, the pressure distributions and cannula jet stagnation region severity will be presented. The second half of the Results Section will focus entirely on cerebral blood flow and comparative cerebral hemodynamics of the two cannula configurations.

\subsection{Effect of adding cerebral arteries to the aortic arch geometry}

The total flow rate that is perfused to the brain is computed to be the same for both the truncated aortic arch and the integrated-CoW arch model as expected. However the flow distributions at the individual head-neck arteries are found to be substantially different. For 
Piskin, S. et al: Computational modeling of neonatal cardiopulmonary bypass

the integrated model with CoW, cerebral perfusion is achieved primarily through SA branch instead of the IA as in the isolated aorta model. For the isolated aorta geometry with DAcannula configuration, the percent (\%) flows splits were computed as 23, 9 and 14 for IA, LCA, and SA, respectively. The corresponding percent flow splits for the integrated model are as 15, 11 and 18 for IA, LCA and SA respectively. The streamline patterns and vorticity magnitudes are found to be different for both models except for the narrow DA region. In this section, the comparison of isolated aorta and the integrated model with CoW is illustrated for the DA-cannula configuration but conclusions are similar for the IA-cannula, which is omitted for brevity.

\subsection{Blood streamlines and velocity distribution at aortic arch}

The blood flow pathlines of both cannula configurations are displayed in Figure 3. For both cases a brief developing jet is observed proximal to the cannula tip. Due to smaller neonatal lumen space this jet could not develop fully and stagnated on the vessel wall. Recirculation zones that may contribute to hemolysis are observed at the IA and at aortic arch - head neck vessel anastomosis region for IA-cannula configuration while these regions are stayed laminar for DA-cannula configuration. These vortices are generated due to the flow exiting the cannula, which hits directly to the IA wall, and splits there, before continuing its more laminar flow. To improve hemodynamics, the IA-cannula can be inclined slightly with respect to the vessel wall, which will decrease the vortices at IA and aortic arch -head neck vessel anastomosis region significantly. DA-cannula configuration also displayed unique flow recirculation zones at the PAs proximal to the DA. While the vortex strength is not as strong as the recirculation in IA-cannula configuration, these regions also require attention. Particularly when an extension graft is anastomosed to the PA this flow feature is most severe and can be reduced depending on the extension of the cannula tip in to the DA. 
Piskin, S. et al: Computational modeling of neonatal cardiopulmonary bypass

Velocity vectors and velocity magnitudes are plotted for the full geometry and for the arch region in Figure 3. It is observed that for DA-cannula configuration, the maximum velocity occurs at the cannula just before exit after the velocity profile is fully developed as expected. For IA-cannula configuration, the maximum velocity occurs at the mid aorta sections closer to the exit of cannula and IA anastomosis location. The maximum velocity at aortic arch -head neck vessel anastomosis region is higher than that of at cannula profile. Maximum velocity of IA-cannula configuration can be reduced by changing the inclination angle of cannula or diameter. DA-cannula configuration has lower velocity magnitudes in general than the configuration employing IA-cannula..

\subsection{Blood stagnation, pressure, wall shear stress and energy loss}

Pressure distribution at the arch region is plotted in Figure 4, highlighting the cannula jet stagnation pressure severity. Figure 4 presents the pressure distribution at aortic arch region while focusing on the pressure distribution at stagnation regions across the end of the cannulas. DA-cannula configuration resulted higher local pressure values compared to IAcannula configuration while ventricle outlet pressures for IA and DA-cannula configurations are similar. Pressure values at stagnation regions of both configurations are $33 \mathrm{mmHg}$ and 34 $\mathrm{mmHg}$, respectively. Those regions have higher pressure values than the rest of the geometry for both configurations.

Definition of a single quantitative cannula pressure drop value is not straightforward due to the complex neonatal arch system and external jet flow. Therefore in Table 4 the pressure drop values along main flow segments are tabulated for both configurations together with their percentage difference. Pressure drop is measured between each outlet and cannula inlet. IA-cannula configuration has higher pressure drop for all outlets compared to DAcannula configuration. This is consistent with the total energy dissipation values [29-32], 
Piskin, S. et al: Computational modeling of neonatal cardiopulmonary bypass

which are $7.7 \mathrm{~Pa} \cdot \mathrm{Kg} / \mathrm{s}$ and $5.8 \mathrm{~Pa} \cdot \mathrm{Kg} / \mathrm{s}$ for IA- and DA-cannula configuration, respectively. Highest pressure drops are at ascending aorta and subclavian artery for IA-cannula configuration while they are at ascending aorta and right anterior cerebral artery for DAcannula configuration. Maximum difference of pressure drop between cases occurs at descending aorta with $50 \%$.

Wall Shear Stress (WSS) distribution at cannula and aortic arch and stagnation regions are given in Figures 5. As such, WSS distribution is generally higher for IA-cannula configuration with respect to DA-cannula configuration and consistent with higher energy dissipation trend of IA-cannula configuration. Maximum WSS values are around cannula and stagnation region across the cannula connection for both cases. WSS values are around $40 \mathrm{~Pa}$ and $15 \mathrm{~Pa}$ for IA- and DA-cannula configuration, respectively. For IA-cannula configuration, the connection between IA and aortic arch -head neck vessel anastomosis region has the highest WSS due to diverging shape and high velocity gradients. WSS at stagnation region of IA-cannula configuration is around $40 \mathrm{~Pa}$ and $10 \mathrm{~Pa}$ for stagnation region of DA-cannula configuration. Difference between WSS values of both cases at stagnation region is about $300 \%$.

\subsection{Systemic and cerebral perfusion}

Flow perfusion and flow split to systemic and cerebral arteries are summarized in Table 3 for the two cannula configurations studied. Flow splits for the integrated model with IA-cannula configuration at neck arteries compared to the integrated model with DA-cannula configuration are $40 \%,-45 \%$, and $56 \%$ different for SA, IA and LCA, respectively. Larger differences if flow-splits are observed for Aao and cerebral arteries reaching 200\% and 3090\% respectively. Likewise, the ascending aorta flow rate, which feeds coronary arteries for the hypoplastic left heart, is approximately 3 times greater for IA-cannula configuration 
Piskin, S. et al: Computational modeling of neonatal cardiopulmonary bypass

compared to DA-cannula configuration. The least difference between cannula configurations is computed for the left subclavian artery to be $8 \%$. The change for Dao flow is $\sim 19 \%$ which is also a substantial difference for systemic flow.

Cerebral perfusion is improved for the IA-cannula configuration, about 30 to $90 \%$, while the flow rates at the descending aorta and SA are $20 \%$ and $8 \%$ lower, respectively for IA-cannula configuration compared to DA-cannula configuration. There is another interesting finding presented in Table 3 with regard to symmetric flow at brain artery outlets although the resistance values at left and right cerebral arteries are assigned the same. The flow rates at right and left cerebral arteries are very close to each other in the IA-cannula configuration (i. e. RACA flow rate is close to LACA flow rate) indicating a balanced and symmetrical cerebral perfusion for the IA-cannula configuration. In contrast for the DA-cannula configuration the cerebral perfusion is unbalanced between the anterior and middle cerebral lobes where the differences between the RACA and LACA reach $16 \%$.

Therefore it can be concluded that in general an IA-cannula configuration is better for coronary and cerebral perfusions and leads balanced flow split at brain arteries acutely.

\subsection{Flow redistribution in the Circle of Willis}

Flow rates at interior cerebral arteries are found to be significantly different for the two cannula configurations studied. Figure 6 shows these differences as percentage at right and left posterior communicating arteries and the connecting arteries between RACA and RMCA and LACA and LMCA. Computed differences for the communicating arteries is almost 50\% and less for connecting arteries. Therefore, for different cannula configurations the interior cerebral flows also have major differences as observed at the cerebral outlets. This is important to estimate the ideal blood carrying capacity of the specific communicating and 
Piskin, S. et al: Computational modeling of neonatal cardiopulmonary bypass

connecting arteries. When one of the arteries is absent congenitally or underdeveloped, other communicating or connecting arteries can take over the necessary blood perfusion.

Figure 7 compares the WSS distributions of both cases by focusing on heard-neck and cerebral arteries. IA-cannula configuration has generally higher WSS values at the left-sided arteries while an opposite trend is observed for the DA-cannula configuration. For the headneck arteries, IA-cannula configuration resulted lower WSS values. DA-cannula configuration has symmetrical WSS distribution at left and right cerebral and head-neck arteries while non-symmetrical WSS distribution is computed for the IA-cannula configuration. These trends in WSS loading is consistent with findings reported for flowsplits.

\subsection{Flow reversal at the head neck arteries}

In Figure 6 the 3D distribution of velocity vectors are plotted for the head-neck and interior cerebral arteries for both configurations. These plots confirm that the IA-cannula configuration generally result higher velocity magnitudes compared to the DA-cannula configuration. DA-cannula configuration has symmetrical velocity vector distribution at left and right neck and interior cerebral arteries while IA-cannula configuration does not. Left interior arteries have lower velocity magnitude compared to right arteries for IA-cannula. This is an interesting result since the flow splits for IA-cannula configuration are symmetrical in terms of left and right cerebral outlets while it is nonsymmetrical for DA-cannula configuration. This suggests that the perfusion is symmetrical for IA-cannula configuration even though the interior flow is not. Another interesting finding is related to the natural flow direction through the downstream head-neck arteries. For the DA-cannula configuration flow is consistently towards the head, while for the IA-configuration it is reversed at LVA vessel, Figure 6. 
Piskin, S. et al: Computational modeling of neonatal cardiopulmonary bypass

\section{Discussion}

In adult CPB, intra-operative outflow cannula positioning [33] has been shown to have clinically significant impact in mitigating clinical stroke-risk [34]. Whereas for neonatal bypass, the earlier 3D models of neonatal aortic arch that terminate at the head-neck vessels was inconclusive to quantify the influence of cannula position due to high peripheral vascular resistance boundary conditions. The present $3 \mathrm{D}$ model that includes the full cerebral arterial system reported significant differences in blood flow distribution to the major cerebral lobes for different cannula configurations, intraoperatively. While these results need to be confirmed through in vivo animal experiments as well as appropriately designed clinical studies, present comparative study illustrates that neonatal cannula alters cerebral hemodynamics in the infant brain and quantifies the expected differences. It should be emphasized that present results correspond to the acute changes in hemodynamics, which can be toned-down in vivo, through autoregulation and other physiological factors, including the vessel compliance and pulsatility.

Computed WSS distribution at the aortic arch for IA-cannula configuration was compared with our previous studies [9] for validation. IA-cannula configuration was selected for validation since it resembles the previous configuration more closely. Both studies indicated that the highest WSS occurred at the cannula stagnation region across cannula connection. For both studies, WSS distributions are similar at aortic arch and subclavian artery. However, maximum WSS for previous study was about $130 \mathrm{~Pa}$ while it is about $40 \mathrm{~Pa}$ for current study due to differences in the cannula sizes. Also, the flow splits at neck arteries change substantially after inclusion of neck and cerebral arteries to aortic arch geometry.

A major issue in the CPB is the development of thrombosis associated with the platelet-activation, red blood cell damage under the highly turbulent cannula jet flow. Blood damage performance and the risk of thrombosis formation of the cannula strategy can be 
Piskin, S. et al: Computational modeling of neonatal cardiopulmonary bypass

assessed using the present numerical model but postponed to future communications. Still, recirculation zones that may contribute to hemolysis are observed at the IA and at aortic arch -head neck vessel anastomosis region for IA-cannula configuration while these regions are stayed laminar for DA-cannula configuration. To improve hemodynamics the IA-cannula can be inclined slightly with respect to the wall, which will decrease the vortices. DA-cannula configuration also displayed unique flow recirculation zones at the PAs proximal to the DA. Particularly for cases when an extension graft is anastomosed to the PA this flow feature will be most severe and can be reduced by controlling the extension of the cannula tip in to the DA. Also, the IA-cannula configuration has generally higher velocity at especially right neck and cerebral arteries (such as RCA, RVA, RACA), which could cause blood damage locally at those locations.

Subject-to-subject variability of congenital heart defects and complex anatomies results a large spectrum of cannula and circuit configurations that are adopted due to the preference of individual surgeon $[10,35]$. Present work tested the two of the common configurations on a realistic congenital heart disease template. For further improvement, present methodology can be expanded to include myocardial tissue beds, patient-specific anatomies as well as missing CoW branches. Additional configurations that can be studied through the present modeling approach include; double cannula configurations used in interrupted aortic arch, dilator cannula or dilation grafts sewn typically on the innominate artery. Parameters that can be varied and influence CPB hemodynamics but not considered in the present study include the insertion distance between the cannula tip and the inner wall, use of use a clamp vs. snare during decanulation process, vacuum-assisted venous return, reperfusion strategy once the aortic clamp is removed, effect of PA banding, bypass flow rate, blood temperature and rate and depth of cooling. We will analyze the effect of missing anterior or posterior communicating artery. Also the obstruction effect of the three arteries 
Piskin, S. et al: Computational modeling of neonatal cardiopulmonary bypass

that connect the brain artery tree with the aorta (basilar artery or any or both of the carotid arteries) will be investigated. We anticipate that in some configurations flow markedly varies based simply on cannula tip orientation, depth of cannula insertion even changes that are at the order of millimeters can result major differences in hemodynamics.

Difference between WSS values of both cases at stagnation region is about $300 \%$. Change of inclination angle of cannula in IA-cannula configuration towards the aortic arch sinus could decrease WSS by lowering velocity gradients and getting rid of diverging geometry effect. DA-cannula configuration is preferable in terms of lower WSS. Also, the hypo plastic ascending aorta has higher WSS than descending aorta and pulmonary arteries for both cases. This is due to very small radius and relatively high flow rate at ascending aorta outlet (going in to coronary arteries).

As shown in Figure 6 there is antegrade at RVA and retrograde flow at LVA. This is called LSA steal from LVA [36]. Horrow et al visualized a similar retrograde flow in case of occlusion or near occlusion of LSA. This implies that the IA-cannula configuration may result LSA occlusion by stealing blood from LVA. Our prototype low-pressure drop cannula diffuser tip design was successful in eliminating the backflow in all cannula positions $[1,6$, 9]. Pressure distribution at neck and cerebral arteries for both cases are shown in Figure 7. DA-cannula configuration has generally higher pressure compared to IA-cannula configuration. DA-cannula configuration has symmetrical pressure distribution at left and right cerebral and neck arteries while IA-cannula configuration does not have symmetrical pressure distribution at left and right cerebral and neck arteries. Left arteries have lower pressure values compared to right arteries for IA-cannula configuration. Cannula configuration has also effect on symmetrical or nonsymmetrical flow splits in terms of left and right neck and cerebral arteries. Interior and outflow distributions depend on the cannula location and angle. 
Piskin, S. et al: Computational modeling of neonatal cardiopulmonary bypass

Our results indicate that each cannula prototype has an optimal disease-specific intraoperative configuration that will maximize its cerebral perfusion and optimize the competing cerebral and myocardial flow demands during reperfusion, while minimizing blood damage.

\section{Conclusions}

3D-0D coupled simulation of hypoplastic neonatal aortic arch with cerebral arteries during CPB procedure was realized in this study. Two different cannula configurations were simulated and compared to each other. Results suggest that flow distributions at main outlets depend significantly on cannula configuration as well as the peripheral resistance values. Inclusion of the cerebral arteries influences the results compared to the model with an isolated aortic arch. Cannula configuration has substantial effect on local hemodynamics including the flow path lines, velocity magnitude, stagnation pressure and WSS levels. To our knowledge present work is the first model that describes detailed cerebral arterial flow dynamics during neonatal bypass. In general results favored an IA-cannula configuration compared to a DA-cannula for hypoplastic arch reconstruction in terms of higher coronary perfusion and symmetric cerebral perfusion.

\section{Acknowledgements}

EU FP7 293931- CardioFluidMechanics and ERC STR Grant 307460 to KP. 
Piskin, S. et al: Computational modeling of neonatal cardiopulmonary bypass

\section{References}

[1] P. G. Menon, J. F. Antaki, A. Undar, and K. Pekkan, "Aortic outflow cannula tip design and orientation impacts cerebral perfusion during pediatric cardiopulmonary bypass procedures," Ann Biomed Eng, vol. 41, pp. 2588-602, Dec 2013.

[2] T. A. Kaufmann, P. Schlanstein, A. Moritz, and U. Steinseifer, "Development of a Hemodynamically Optimized Outflow Cannula for Cardiopulmonary Bypass," Artif Organs, Feb 172014

[3] M. Neidlin, S. Jansen, A. Moritz, U. Steinseifer, and T. A. Kaufmann, "Design Modifications and Computational Fluid Dynamic Analysis of an Outflow Cannula for Cardiopulmonary Bypass," Ann Biomed Eng, Jul 122014.

[4] I. Avrahami, B. Dilmoney, A. Azuri, M. Brand, O. Cohen, L. Shani, et al., "Investigation of risks for cerebral embolism associated with the hemodynamics of cardiopulmonary bypass cannula: a numerical model," Artif Organs, vol. 37, pp. 85765, Oct 2013.

[5] I. Avrahami, B. Dilmoney, O. Hirshorn, M. Brand, O. Cohen, L. Shani, et al., "Numerical investigation of a novel aortic cannula aimed at reducing cerebral embolism during cardiovascular bypass surgery," J Biomech, vol. 46, pp. 354-61, Jan 182013.

[6] P. G. Menon, N. Teslovich, C. Y. Chen, A. Undar, and K. Pekkan, "Characterization of neonatal aortic cannula jet flow regimes for improved cardiopulmonary bypass," $J$ Biomech, vol. 46, pp. 362-72, Jan 182013.

[7] T. A. Kaufmann, M. Hormes, M. Laumen, D. L. Timms, T. Linde, T. Schmitz-Rode, et al., "The impact of aortic/subclavian outflow cannulation for cardiopulmonary bypass and cardiac support: a computational fluid dynamics study," Artif Organs, vol. 33, pp. 727-32, Sep 2009.

[8] T. A. Kaufmann, M. Hormes, M. Laumen, D. L. Timms, T. Schmitz-Rode, A. Moritz, et al., "Flow distribution during cardiopulmonary bypass in dependency on the outflow cannula positioning," Artif Organs, vol. 33, pp. 988-92, Nov 2009.

[9] K. Pekkan, O. Dur, K. Sundareswaran, K. Kanter, M. Fogel, A. Yoganathan, et al., "Neonatal aortic arch hemodynamics and perfusion during cardiopulmonary bypass," J Biomech Eng, vol. 130, p. 061012, Dec 2008.

[10] D. de Zélicourt, P. Jung, M. Horner, K. Pekkan, K. R. Kanter, and A. P. Yoganathan, "Cannulation Strategy for Aortic Arch Reconstruction Using Deep Hypothermic Circulatory Arrest," The Annals of Thoracic Surgery, vol. 94, pp. 614-620, 8// 2012.

[11] T. A. Kaufmann, K. C. Wong, T. Schmitz-Rode, and U. Steinseifer, "Mimicking of cerebral autoregulation by flow-dependent cerebrovascular resistance: a feasibility study," Artif Organs, vol. 36, pp. E97-101, Apr 2012.

[12] T. A. Kaufmann, M. Neidlin, M. Busen, S. J. Sonntag, and U. Steinseifer, "Implementation of intrinsic lumped parameter modeling into computational fluid dynamics studies of cardiopulmonary bypass," J Biomech, vol. 47, pp. 729-35, Feb 7 2014.

[13] M. Neidlin, U. Steinseifer, and T. A. Kaufmann, "A multiscale 0-D/3-D approach to patient-specific adaptation of a cerebral autoregulation model for computational fluid dynamics studies of cardiopulmonary bypass," J Biomech, vol. 47, pp. 1777-83, Jun 3 2014.

[14] P. Reorowicz, D. Obidowski, P. Klosinski, W. Szubert, L. Stefanczyk, and K. Jozwik, "Numerical simulations of the blood flow in the patient-specific arterial cerebral circle region," J Biomech, vol. 47, pp. 1642-51, May 72014. 
Piskin, S. et al: Computational modeling of neonatal cardiopulmonary bypass

[15] L. Grinberg, E. Cheever, T. Anor, J. R. Madsen, and G. E. Karniadakis, "Modeling blood flow circulation in intracranial arterial networks: a comparative 3D/1D simulation study," Ann Biomed Eng, vol. 39, pp. 297-309, Jan 2011.

[16] N. Hecht, I. Fiss, S. Wolf, M. Barth, P. Vajkoczy, and J. Woitzik, "Modified flowand oxygen-related autoregulation indices for continuous monitoring of cerebral autoregulation," Journal of Neuroscience Methods, vol. 201, pp. 399-403, 10/15/ 2011.

[17] H. Shimizu, T. Matayoshi, M. Morita, T. Ueda, and R. Yozu, "Total Arch Replacement Under Flow Monitoring During Selective Cerebral Perfusion Using a Single Pump," The Annals of Thoracic Surgery, vol. 95, pp. 29-34, 1// 2013.

[18] J. Steinbrink, T. Fischer, H. Kuppe, R. Hetzer, K. Uludag, H. Obrig, et al., "Relevance of depth resolution for cerebral blood flow monitoring by near-infrared spectroscopic bolus tracking during cardiopulmonary bypass," The Journal of Thoracic and Cardiovascular Surgery, vol. 132, pp. 1172-1178, 11// 2006.

[19] T. Abramo, N. Aggarwal, I. Kane, K. Crossman, and M. Meredith, "Cerebral oximetry and cerebral blood flow monitoring in 2 pediatric survivors with out-ofhospital cardiac arrest," The American Journal of Emergency Medicine, vol. 32, pp. 394.e5-394.e10, 4// 2014.

[20] J. C. Hirsch, M. L. Jacobs, D. Andropoulos, E. H. Austin, J. P. Jacobs, D. J. Licht, et al., "Protecting the Infant Brain During Cardiac Surgery: A Systematic Review," The Annals of Thoracic Surgery, vol. 94, pp. 1365-1373, 10// 2012.

[21] S. P. Talpahewa, R. Ascione, G. D. Angelini, and A. T. Lovell, "Cerebral cortical oxygenation changes during OPCAB surgery," The Annals of Thoracic Surgery, vol. 76, pp. 1516-1522, 11// 2003.

[22] B. A. Johnson, G. M. Hoffman, J. S. Tweddell, J. R. Cava, M. Basir, M. E. Mitchell, et al., "Near-Infrared Spectroscopy in Neonates Before Palliation of Hypoplastic Left Heart Syndrome," The Annals of Thoracic Surgery, vol. 87, pp. 571-579, 2// 2009.

[23] O. Dur, S. T. Coskun, K. O. Coskun, D. Frakes, L. B. Kara, and K. Pekkan, "Computer-Aided Patient-Specific Coronary Artery Graft Design Improvements Using CFD Coupled Shape Optimizer," Cardiovasc Eng Technol, vol. 2, pp. 35-47, Mar 2011.

[24] L. Antiga, B. Ene-Iordache, L. Caverni, G. P. Cornalba, and A. Remuzzi, "Geometric reconstruction for computational mesh generation of arterial bifurcations from CT angiography," Computerized Medical Imaging and Graphics, vol. 26, pp. 227-235, 2002.

[25] M. Yoshida, P. D. Wearden, O. Dur, K. Pekkan, and V. O. Morell, "Right Ventricular Outflow Tract Reconstruction With Bicuspid Valved Polytetrafluoroethylene Conduit," The Annals of Thoracic Surgery, vol. 91, pp. 1235-1239.

[26] W. J. Kowalski, O. Dur, Y. Wang, M. J. Patrick, J. P. Tinney, B. B. Keller, et al., "Critical Transitions in Early Embryonic Aortic Arch Patterning and Hemodynamics," PLoS ONE, vol. 8, p. e60271, 2013.

[27] E. Kocyildirim, O. Dur, O. Soran, E. Tuzun, M. W. Miller, G. J. Housler, et al., "Pulsatile venous waveform quality in Fontan circulation-clinical implications, venous assists options and the future," Anadolu Kardiyol Derg, vol. 12, pp. 420-6, Aug 2012.

[28] S. Prakash and C. R. Ethier, "Requirements for mesh resolution in 3D computational hemodynamics," Journal of Biomechanical Engineering, vol. 123, pp. 134-144, 2001.

[29] L. P. Dasi, K. Pekkan, D. de Zelicourt, K. S. Sundareswaran, R. Krishnankutty, P. J. Delnido, et al., "Hemodynamic energy dissipation in the cardiovascular system: 
Piskin, S. et al: Computational modeling of neonatal cardiopulmonary bypass

generalized theoretical analysis on disease states," Ann Biomed Eng, vol. 37, pp. 66173, Apr 2009.

[30] J. Chung, K. Lachapelle, E. Wener, R. Cartier, B. De Varennes, R. Fraser, et al., "Energy loss, a novel biomechanical parameter, correlates with aortic aneurysm size and histopathologic findings," The Journal of Thoracic and Cardiovascular Surgery, vol. 148, pp. 1082-1089, 9// 2014.

[31] C. W. Akins, B. Travis, and A. P. Yoganathan, "Energy loss for evaluating heart valve performance," The Journal of Thoracic and Cardiovascular Surgery, vol. 136, pp. 820-833, 10// 2008.

[32] T. Bottio, C. Dal Lin, A. Lika, G. Rizzoli, V. Tarzia, E. Buratto, et al., "In vitro comparison of different mechanical prostheses suitable for replacement of the systemic atrioventricular valve in children," The Journal of Thoracic and Cardiovascular Surgery, vol. 143, pp. 558-568, 3// 2012.

[33] A. F. Osorio, R. Osorio, A. Ceballos, R. Tran, W. Clark, E. A. Divo, et al., "Computational fluid dynamics analysis of surgical adjustment of left ventricular assist device implantation to minimise stroke risk," Comput Methods Biomech Biomed Engin, vol. 16, pp. 622-38, 2013.

[34] D. B. Andropoulos, K. M. Brady, R. B. Easley, and C. D. Fraser, Jr., "Neuroprotection in Pediatric Cardiac Surgery: What is On the Horizon?," Prog Pediatr Cardiol, vol. 29, pp. 113-122, Aug 12010.

[35] G. Derk, H. Laks, R. Biniwale, S. Patel, K. De LaCruz, E. Mazor, et al., "Novel techniques of mechanical circulatory support for the right heart and Fontan circulation," Int J Cardiol, Aug 82014.

[36] M. M. Horrow and J. Stassi, "Sonography of the Vertebral Arteries," American Journal of Roentgenology, vol. 177, pp. 53-59, 2001/07/01 2001. 
Piskin, S. et al: Computational modeling of neonatal cardiopulmonary bypass

Table 1: List of abbreviations of the major arteries investigated. Arteries are grouped into three lists; Arteries at aortic arch, arteries belong to neck region and arteries belong to cerebral region.

\begin{tabular}{lll} 
Abbreviation & Long Name & BC Type \\
\hline & \multicolumn{1}{c}{ Arteries at Aortic Arch } \\
Aao & Ascending Aorta & Outlet \\
Ao & Aorta & Internal \\
DA & Ductus Arteriosus & Internal \\
PDA & Patent Ductus Arteriosus & Internal \\
Dao & Descending Aorta & Outlet \\
LPA & Left Pulmonary Artery & Wall \\
RPA & Right Pulmonary Artery & Wall \\
MPA & Main Pulmonary Artery & Wall \\
& Arteries belong to Neck Region & \\
IA & Brachiocephalic (Innominate) Artery & Internal \\
BaA & Basilar Artery & Internal \\
SA & (Left) Subclavian Artery & Outlet \\
LCA & Left Carotid Artery & Internal \\
RCA & Right Carotid Artery & Internal \\
LVA & Left Vertebral Artery & Internal \\
RVA & Right Vertebral Artery & Internal \\
& Arteries belong to Cerebral Region & \\
ACoA & Anterior Communicating Artery & Internal \\
RPCoA & Right Posterior Communicating Artery & Internal \\
LPCoA & Left Posterior Communicating Artery & Internal \\
RACA & Right Anterior Cerebral Artery & Outlet \\
LACA & Left Anterior Cerebral Artery & Outlet \\
RMCA & Right Middle Cerebral Artery & Outlet \\
LMCA & Left Middle Cerebral Artery & Outlet \\
RPCA & Right Posterior Cerebral Artery & Outlet \\
LPCA & Left Posterior Cerebral Artery & Outlet \\
\hline & & \\
& &
\end{tabular}


Piskin, S. et al: Computational modeling of neonatal cardiopulmonary bypass

Table 2: Mesh convergence table shows the element size versus number of nodes.

\begin{tabular}{lcc} 
Name of Case & Element Size $(\mathbf{m m})$ & Number of Nodes \\
\hline Coarser & 1.30 & 188,467 \\
Coarse & 1.00 & 400,094 \\
Regular & 0.70 & $1,135,156$ \\
Fine & 0.55 & $2,304,779$ \\
Finer & 0.50 & $3,055,023$ \\
Finest & 0.45 & $4,168,947$ \\
\hline
\end{tabular}


Piskin, S. et al: Computational modeling of neonatal cardiopulmonary bypass

Table 3: Flow rates and percentages at aorta and artery outlets are given in this table.

Difference is calculated by dividing absolute difference of flow rates to DA-cannula configuration flow rate. Biggest differences occur at Aao (around 200\%) and cerebral arteries (around $30 \%$ to $90 \%$ ). Flow split is defined as ratio of outlet flow rate to total inlet flow rate at cannula. Table also gives the difference of flow rates between two cannula configurations. According to those results, cannula configuration changes flow rates at outlets of arteries substantially.

\begin{tabular}{lccccc} 
& \multicolumn{2}{c}{ IA-cannula Configuration } & \multicolumn{2}{c}{ DA-cannula Configuration } & Difference \\
Artery Name & Flow rate (I/m) & Flow Split (\%) & Flow rate (I/m) & Flow Split (\%) & $\%$ \\
\hline \multicolumn{7}{c}{ Systemic } \\
Cannula & 0.5251 & -100.00 & 0.5207 & -100.00 & 0 \\
Aao & -0.0219 & 4.16 & -0.0073 & 1.40 & 198 \\
Dao & -0.2691 & 51.25 & -0.3293 & 63.25 & 19 \\
MPA & 0.0000 & 0.00 & 0.0000 & 0.00 & 0 \\
LPA & 0.0000 & 0.00 & 0.0000 & 0.00 & 0 \\
RPA & 0.0000 & 0.00 & 0.0000 & 0.00 & 0 \\
SA & -0.0709 & 13.50 & -0.0762 & 14.63 & 8 \\
& & & & \\
LACA & -0.0271 & 5.16 & -0.0160 & 3.07 & 68 \\
LMCA & -0.0273 & 5.21 & -0.0203 & 3.91 & 33 \\
LPCA & -0.0272 & 5.19 & -0.0194 & 3.73 & 39 \\
RACA & -0.0266 & 5.07 & -0.0138 & 2.65 & 91 \\
RMCA & -0.0276 & 5.26 & -0.0185 & 3.56 & 48 \\
RPCA & -0.0273 & 5.21 & -0.0198 & 3.81 & 37 \\
\hline
\end{tabular}


Piskin, S. et al: Computational modeling of neonatal cardiopulmonary bypass

Table 4: Pressure drop values between cannula inlet and aorta and artery outlets are given in this table.

\begin{tabular}{|c|c|c|c|}
\hline Artery Name & $\begin{array}{l}\text { IA-cannula Config. } \\
\text { Pressure drop (mmHg) }\end{array}$ & $\begin{array}{l}\text { DA-cannula Config. } \\
\text { Pressure drop (mmHg) }\end{array}$ & $\begin{array}{c}\text { Difference } \\
\%\end{array}$ \\
\hline \multicolumn{4}{|c|}{ Systemic } \\
\hline Cannula & 0.00 & 0.00 & 0 \\
\hline AA & 10.08 & 10.70 & 6 \\
\hline DA & 6.11 & 4.06 & 50 \\
\hline MPA & 5.97 & 4.69 & 27 \\
\hline LPA & 5.97 & 4.70 & 27 \\
\hline RPA & 5.97 & 4.66 & 28 \\
\hline SA & 6.46 & 4.36 & 48 \\
\hline \multicolumn{4}{|c|}{ Cerebral } \\
\hline LACA & 5.49 & 5.32 & 3 \\
\hline LMCA & 5.56 & 4.82 & 15 \\
\hline LPCA & 5.79 & 5.12 & 13 \\
\hline RACA & 5.72 & 5.72 & 0 \\
\hline RMCA & 5.17 & 5.02 & 3 \\
\hline RPCA & 5.72 & 5.10 & 12 \\
\hline SA & 6.46 & 4.36 & 48 \\
\hline
\end{tabular}


Piskin, S. et al: Computational modeling of neonatal cardiopulmonary bypass

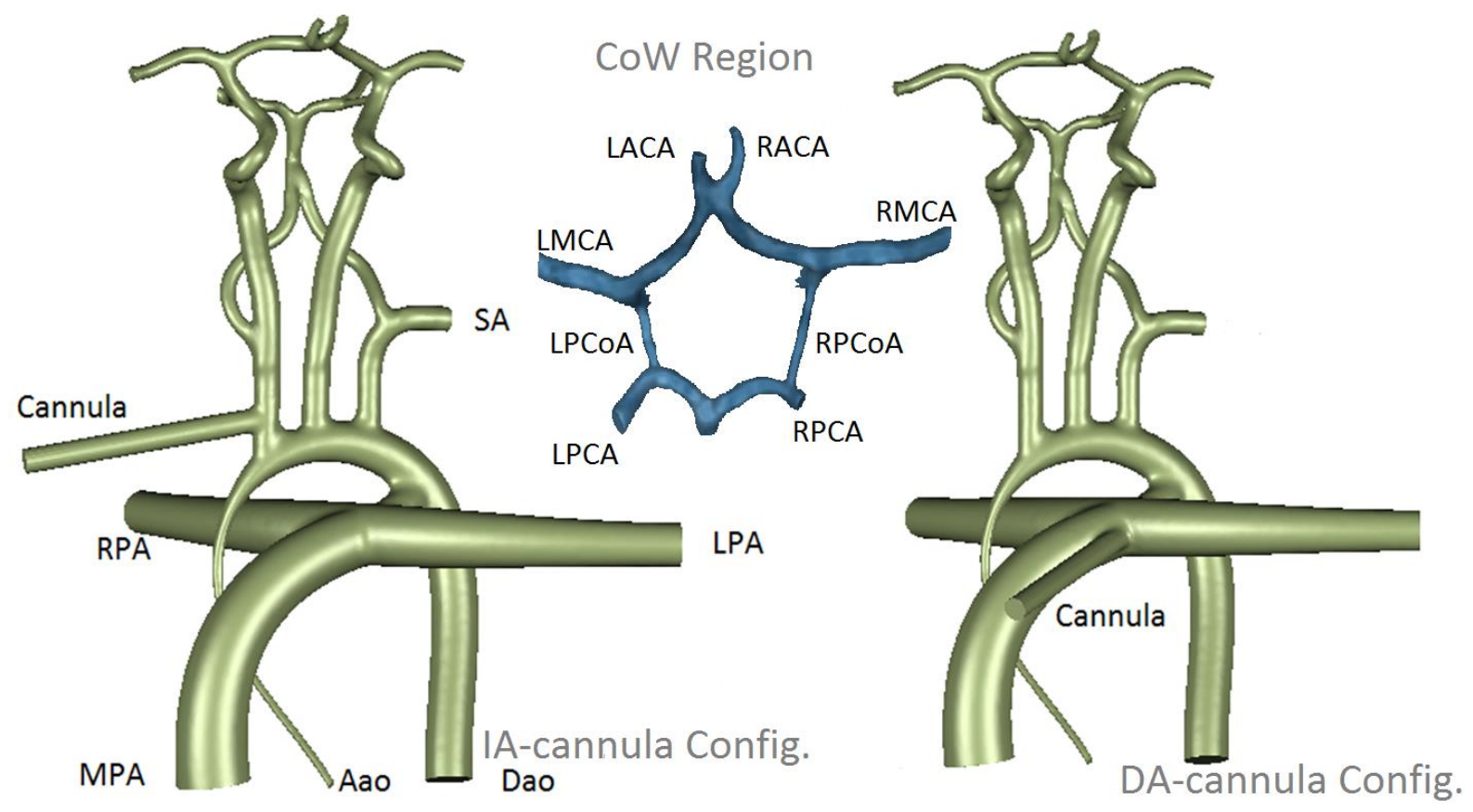

Figure 1: Full geometry model with resistance boundary conditions and zoomed Circle of Willis region. Two different cannula configurations on full geometries: in IA-cannula configuration (left) it is incorporated to Innominate Artery and to Patent Ductus Arteriosus in DA-cannula configuration (right). 
Piskin, S. et al: Computational modeling of neonatal cardiopulmonary bypass
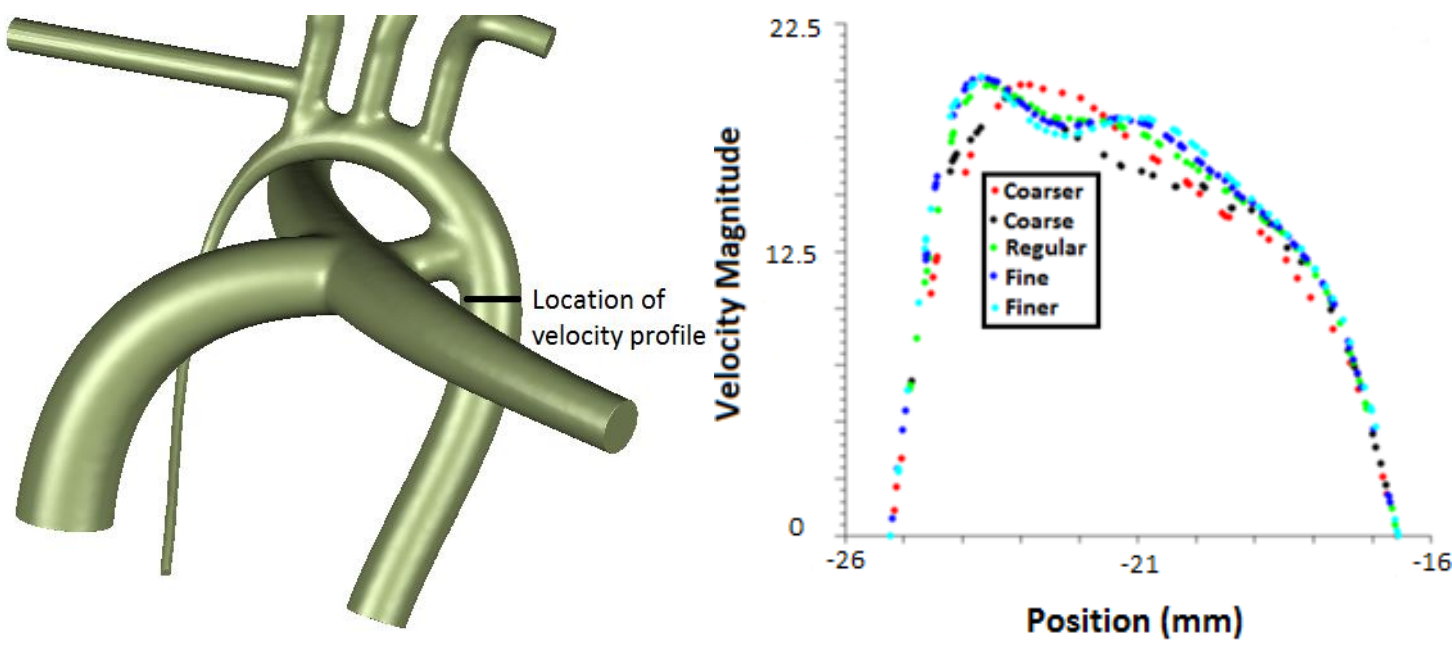

Figure 2: Location of velocity profile (left) and velocity profiles at the selected location. Velocity profiles are obtained for 5 different mesh sizes for mesh convergence test. Regular case is used in all simulations of this study. Velocity profile has an acceptable convergence for mesh sizes smaller than $0.7 \mathrm{~mm}$. They are named as regular, fine and finer meshes in Table 2. 


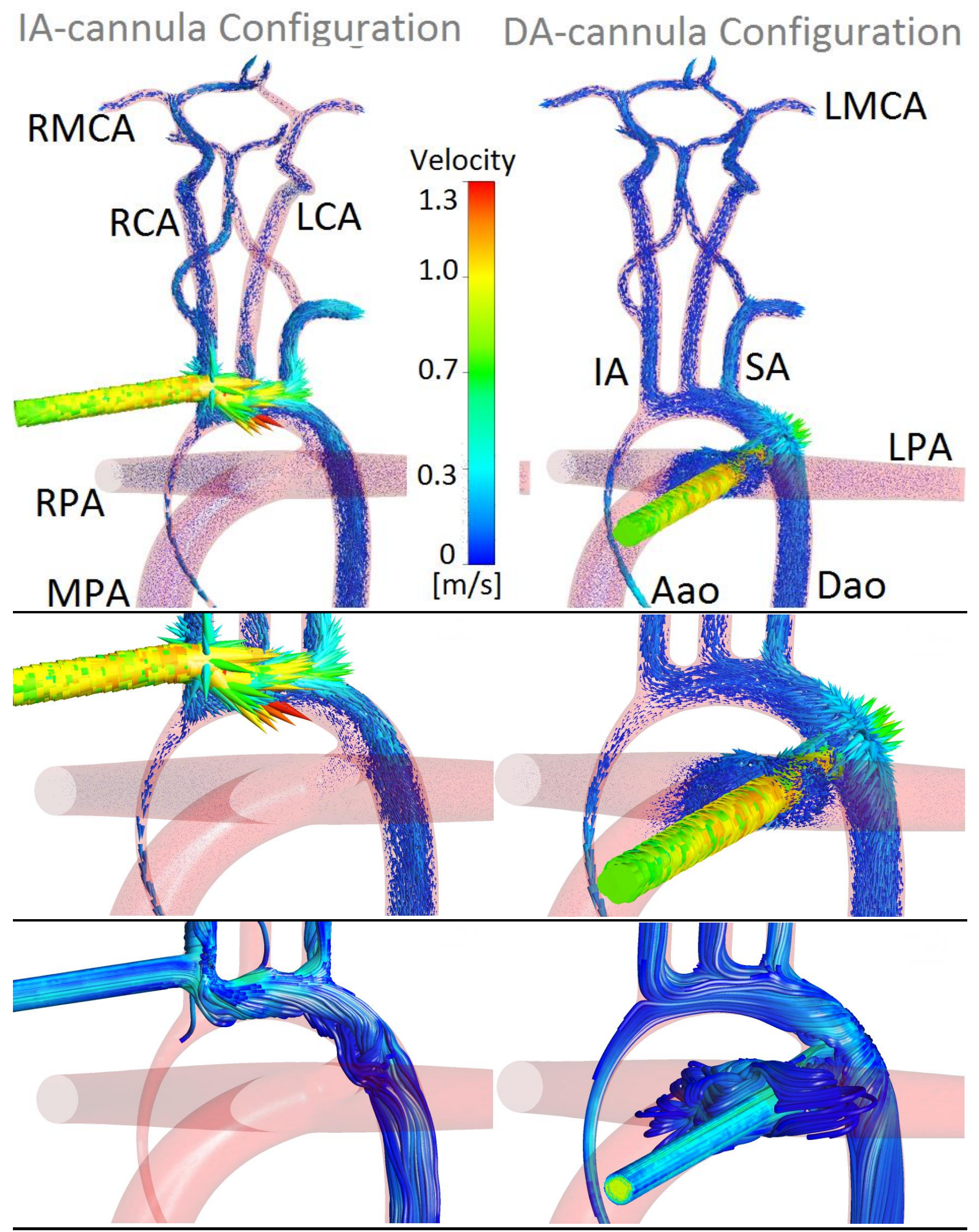

Figure 3: Comparison of velocity vectors and streamlines for two cases with focus (close-up) on aortic arch. 
Piskin, S. et al: Computational modeling of neonatal cardiopulmonary bypass
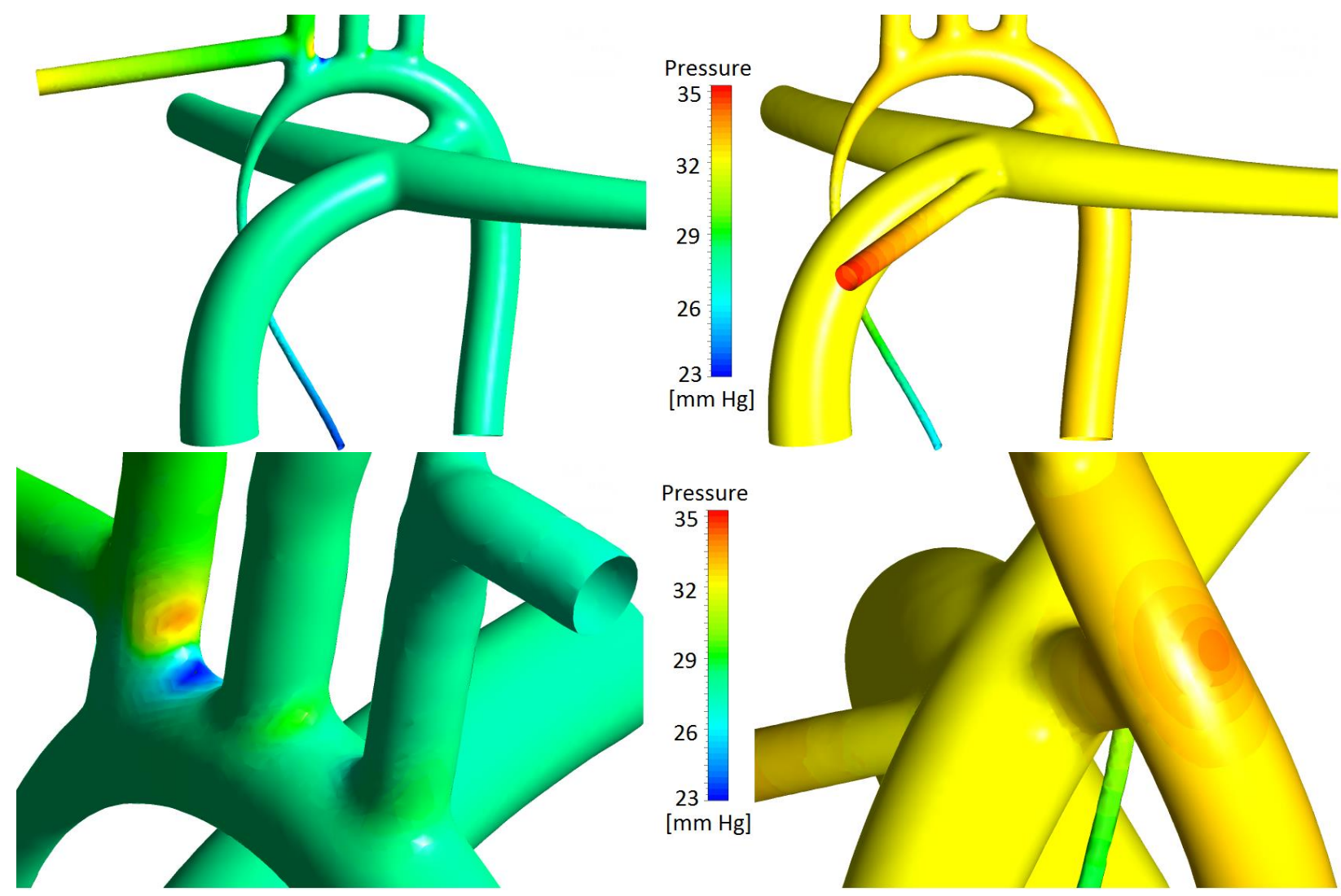

Figure 4: Comparison of pressure distribution for two cases focused on aortic arch and stagnation points. 
Piskin, S. et al: Computational modeling of neonatal cardiopulmonary bypass
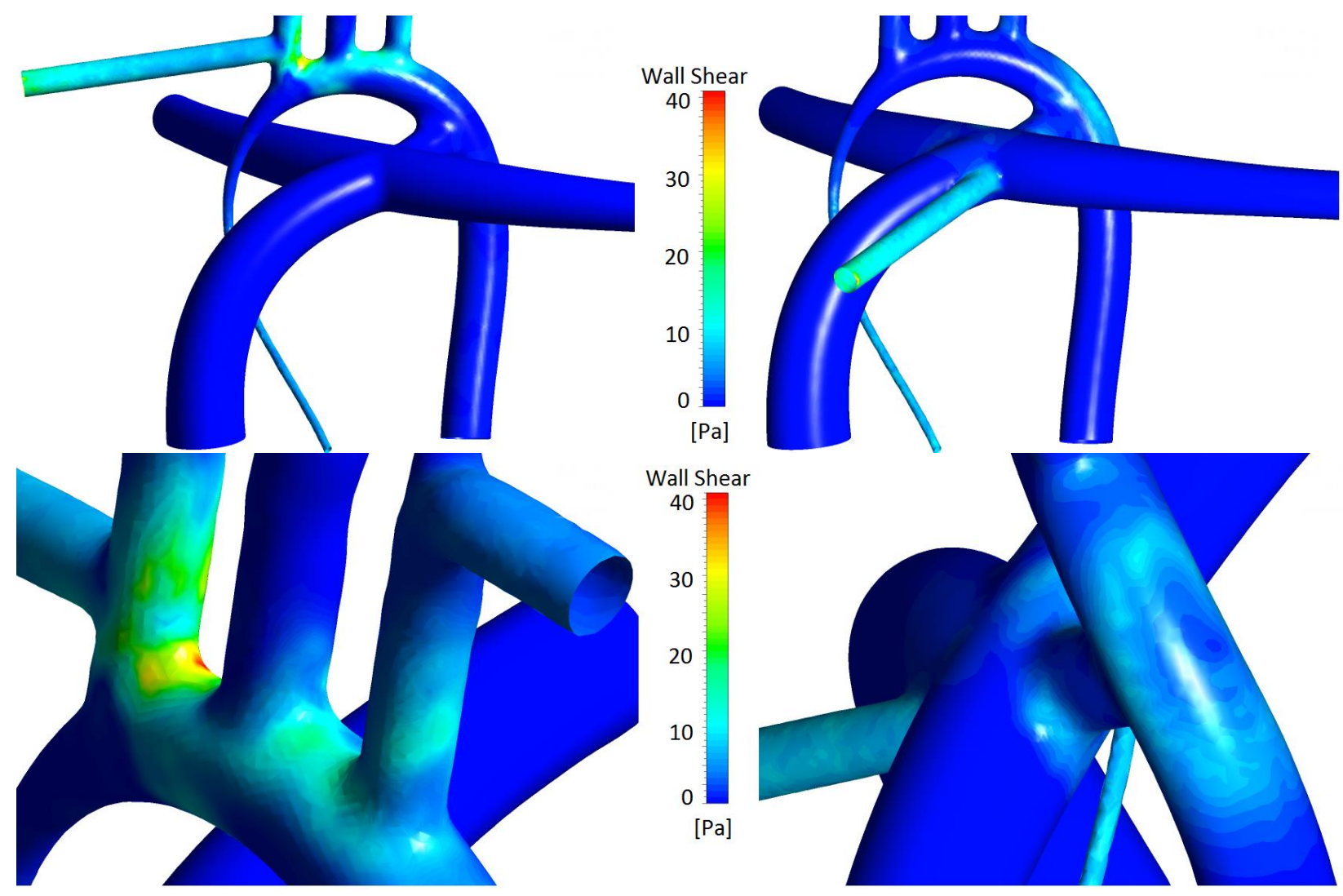

Figure 5: Comparison of WSS distribution for two cases focused on aortic arch. Stagnation regions are indicated by red arrows. 
Piskin, S. et al: Computational modeling of neonatal cardiopulmonary bypass

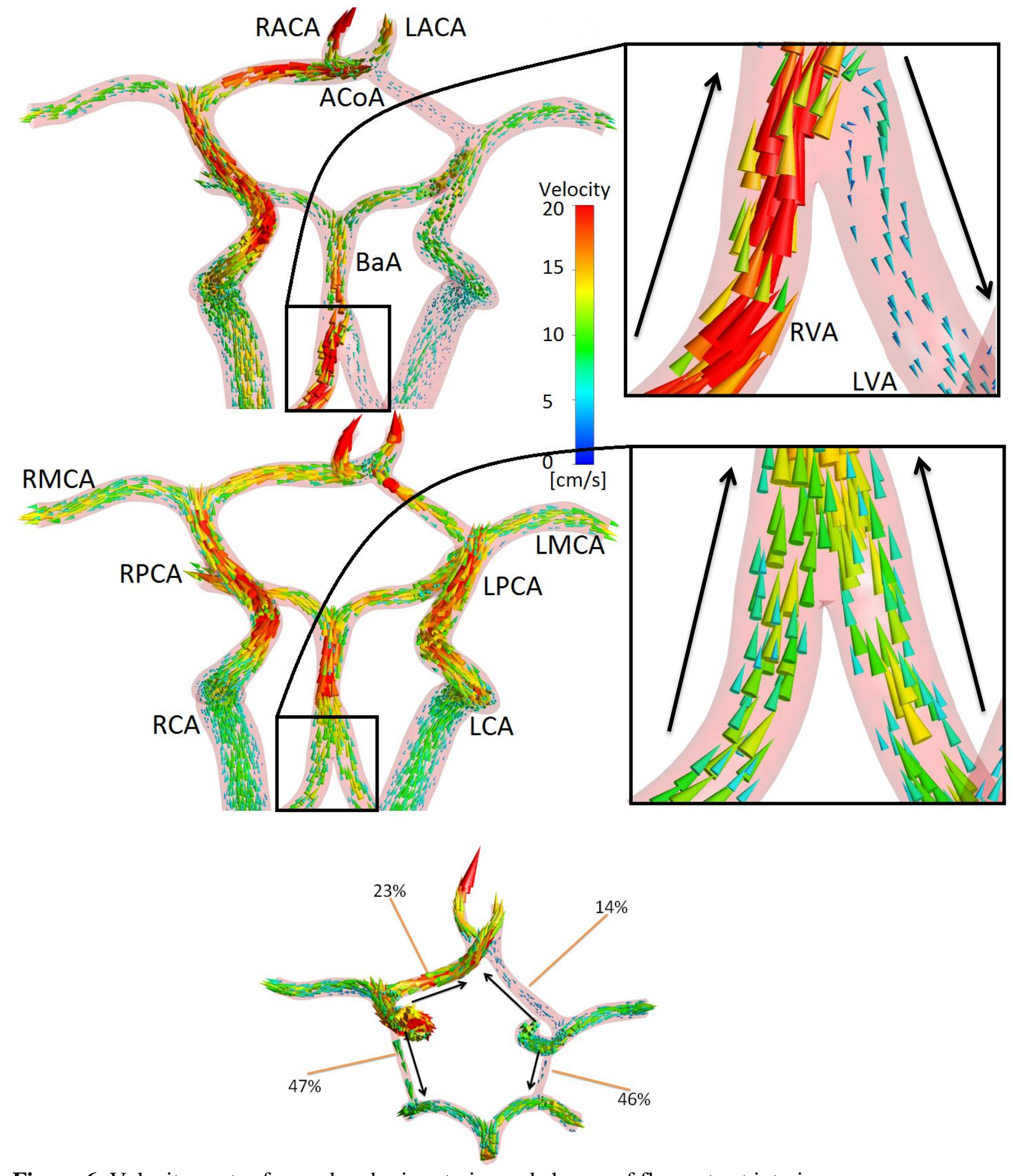

Figure 6: Velocity vector focused on brain arteries and change of flow rate at interior cerebral arteries. 
Piskin, S. et al: Computational modeling of neonatal cardiopulmonary bypass

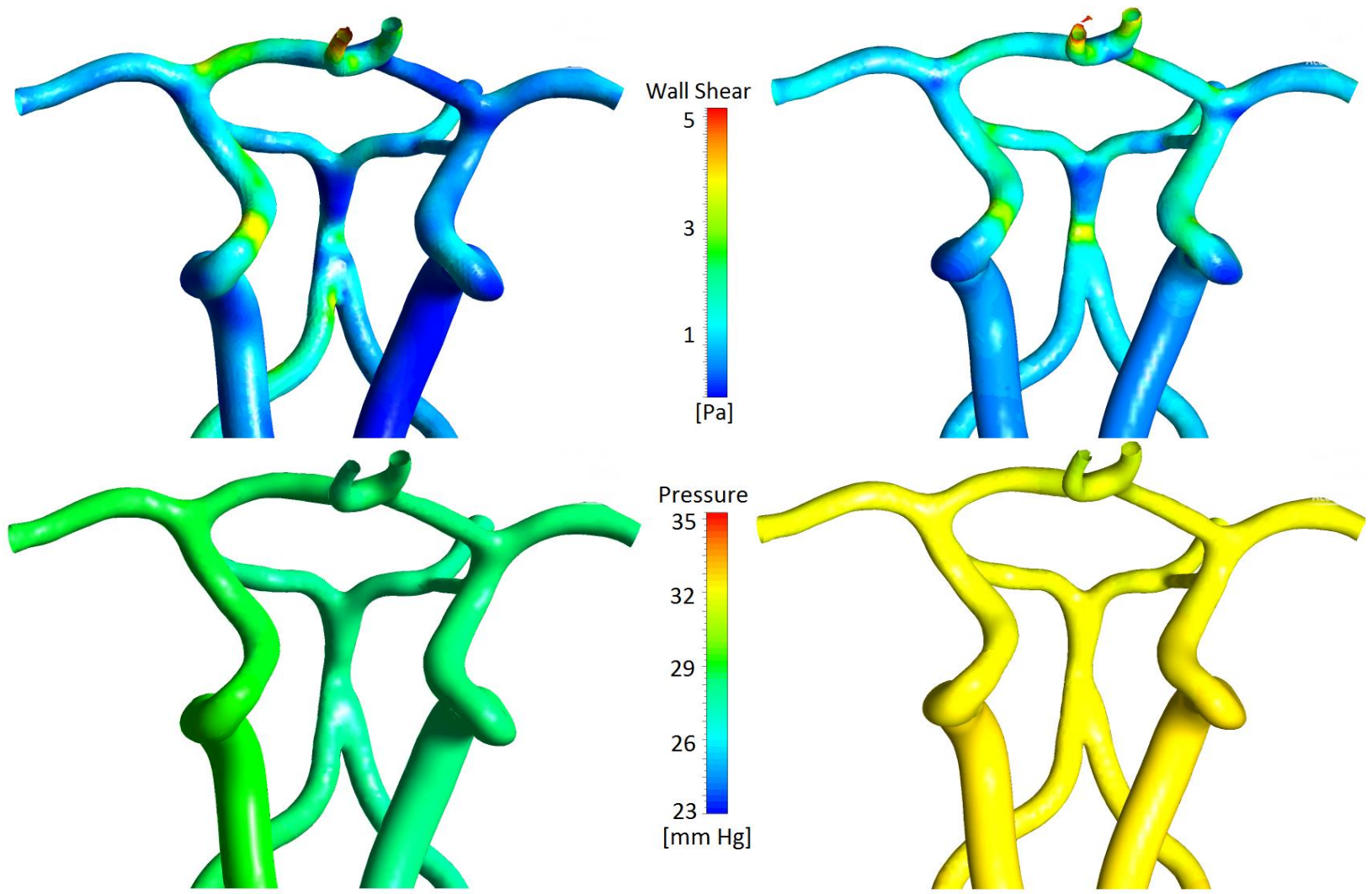

Figure 7: Comparison of WSS and pressure distributions for two cases focused on cerebral arteries. 